DIVISION OF THE HUMANITIES AND SOCIAL SCIENCES

CALIFORNIA INSTITUTE OF TECHNOLOGY

PASADENA, CALIFORNIA 91125

CITIZEN CANDIDATES UNDER UNCERTAINTY

Jon X. Eguia

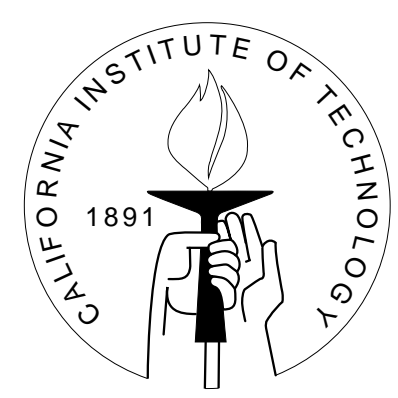

SOCIAL SCIENCE WORKING PAPER 1233

August 2005 


\title{
Citizen Candidates Under Uncertainty
}

\author{
Jon X. Eguia
}

\begin{abstract}
In this paper we make two contributions to the growing literature on "citizen-candidate" models of representative democracy. First, we add uncertainty about the total vote count. We show that in a society with a large electorate, where the outcome of the election is uncertain and where winning candidates receive a large reward from holding office, there will be a two-candidate equilibrium and no equilibria with a single candidate. Second, we introduce a new concept of equilibrium, which we term "sincere-strategic," and we show that with this refinement, the two equilibrium candidates will not be too extreme, one will lean to the left and the other one to the right.
\end{abstract}

JEL classification numbers: D72

Key words: Citizen Candidates, Uncertainty, Strategic Voting, Sincere Voting 


\section{Introduction}

In a representative democracy, citizens elect representatives who in turn, choose policies for the society. Traditional models divide the members of the society into two classes: voters, whose only political role is to vote, and politicians or political parties (often just two of them), who compete in the election. "CitizenCandidate" models of the electoral process, on the other hand, explain how politicians emerge from the class of voters. In these models, some citizens become politicians by choosing to run as candidates in an election. The number and policy preferences of the candidates who run in equilibrium are determined by three factors: the policy preferences of every citizen, the benefits of holding office, and the cost of running for election.

The standard citizen-candidate models suffer from a simplistic assumption that leads to unrealistic predictions. The assumption is that candidates can perfectly anticipate the outcome of the election and forecast exactly how many votes each candidate will receive. This assumption leads to the prediction that two candidates will run against each other only if they have the exact same number of supporters in the electorate. In models with a finite electorate, this implies that typically there will be a two-candidate equilibrium only if the number of citizens is even, because if it is odd, one of the candidates will generally have at least one more supporter than the other.

We solve this problem and assure political competition in the model by introducing uncertainty in the electoral outcome, and then showing that in a society with a large electorate, equilibria with one candidate do not exist, while equilibria with two candidates always exist. We model the uncertainty as in Myerson (1993): Citizens choose which candidate to support, but each citizen has a small probability of failing to convert her intention to support a candidate into an actual valid, counted vote for the candidate -perhaps the voter is unable to make it to the polling station, or she misuses the voting equipment and casts an invalid ballot. This individual probability of being unable to cast a valid vote generates an aggregate uncertainty about the total vote count.

The aggregate uncertainty about the electoral outcome crucially affects elections with a large electorate. Candidates decide to run based on the support they have in the electorate at the time they make the decision to run. However, elections are not deterministic: the aggregate uncertainty makes the outcome stochastic and a candidate who initially had less support may ultimately collect more votes and win the election. If the most popular candidate enters the race, another citizen with slightly less support will also run, in the hope of an upset victory. As a result, in a society with a large enough electorate, one-candidate equilibria do not exist, whereas two-candidate equilibria always exist, regardless of the exact number of citizens. These predictions are similar to those in Osborne and Slivinski's (1996) model of Citizen-Candidates, but Osborne and Slivinski work only with an infinite number of sincere voters, who have rather restricted preferences. Our model, by contrast, allows for any finite number of citizens, who vote strategically and may have quite general forms of preferences.

The uncertainty assumed in our model has a large effect unless the elec- 
torate is very small. In the case of a small electorate (on the order of a few dozens of voters), the predictions of our model are similar to those of Besley and Coate (1997): The existence of equilibria with one or two candidates depends on whether the number of citizens is odd or even.

There are two other papers incorporating uncertainty to a model of citizencandidates: In Riviere's paper (2000), a group of citizens learn their policy preference only after candidacies are announced, so the location of the median is uncertain at the time of the announcement. Our model differs from Riviere's both in the assumptions we use and the results we obtain. In words of the author, Riviere's assumptions are "very restrictive" and in her citizen-candidate model, two-candidate equilibria are "very rare." ${ }^{1}$ Our model relaxes and generalizes most of Riviere's assumptions and shows that under mild conditions, equilibria with two candidates exist.

Roemer (2003) tackles the problem of indeterminacy of equilibria in Besley and Coate's model, advocating a particular refinement (Party-Unanimity Nash Equilibrium or PUNE) that yields a smaller but non-empty set of equilibria. In Roemer's paper, uncertainty is only a side issue. He assumes that each candidate wins with a probability equal to the candidate's share of votes.

We address indeterminacy by offering a criterion to select equilibria: A refinement of our equilibrium concept relaxes the assumption that citizens are strategic in their voting behavior, and requires an equilibrium to hold regardless of whether citizens choose a candidate to support strategically or sincerely. We will show that under this Sincere-Strategic Equilibrium concept, all candidates must receive a similar share of electoral support and that if there are only two, one will lean left and the other one will lean right, but they will not be too extreme.

The rest of the paper is as follows: In Section 2 we present our model of citizen-candidates with uncertainty. In Section 3 we characterize equilibria with one and two candidates and we discuss equilibria with three or more candidates. We summarize the findings of the paper and propose an agenda for future research in Section 4. An Appendix contains technical matters omitted from the text.

\section{The Model}

Let $\mathcal{N}$ be a society formed by $N$ citizens labeled $i \in \mathcal{N}=\{1, \ldots, N\}$. This society must elect a representative, who will receive a benefit $b$ from being elected and will also get to choose a policy in a unidimensional policy space $[0,1]$. The implemented policy we denote by $p$. Citizens have different preferences over the chosen policy; let $v_{i}(p)$ be the utility that citizen $i$ derives from policy $p \in[0,1]$.

Each agent in the society can run as a candidate in the election, but doing so entails a cost $c$, which is small compared to the rewards from holding office.

\footnotetext{
${ }^{1}$ Riviere also considers a game in which like-minded citizens can share the cost of running, forming a political party. In this setting, equilibria with two parties are no longer rare, but for some values of the cost of running, only equilibria with one or three candidates exist.
} 
Formally, we assume that $b>2 c$.

Let $I_{i}=1$ if $i$ runs as a candidate and zero otherwise, and let $W_{i}=1$ if $i$ runs as a candidate and wins the election and zero otherwise.

Then the utility of agent $i$ is:

$$
U\left(p, I_{i}, W_{i}\right)=v_{i}(p)+b * W_{i}-c * I_{i} .
$$

The game has three stages:

In the first stage or entry stage, each citizen simultaneously decides whether or not to enter the race and become a candidate. If no candidate enters the race, then the game ends and a default policy $p_{0}$ is implemented.

In stage two or support stage, each citizen decides to support one of the candidates. Citizens who are indifferent toward all the candidates support no candidate. Each citizen $i$ who supports candidate $j$ casts a valid vote for $j$ with probability $(1-\mu)$. With probability $\mu$, citizen $i$ is unable to cast a valid vote for any candidate, so $i^{\prime} s$ support is lost. This probability $\mu$ is the same for each citizen and it is uncorrelated among citizens. The intuition is that citizen $i$ supports $j$ and intends to vote for $j$, but with probability $\mu$, some reason prevents citizen $i$ from casting a valid vote; perhaps $i$ cannot make it to the polls, or $i$ votes but somehow the ballot is cast incorrectly and is later declared invalid and does not add to the total vote count.

In stage three, once all valid votes are counted, a winner is elected by plurality rule: the candidate who has valid most votes (not necessarily the one with most intended support), wins the election and implements her ideal policy. In case of a tie, the winner is randomly determined among all candidates with most votes.

Citizens know the ideal policy of each candidate and they correctly anticipate that the winner will implement her ideal policy; candidates cannot commit at the entry stage to implement any other policy but their favorite one if they win the election. Empirical evidence indicates that, indeed, voters merely elect the policy they prefer among those offered by the competing candidates, without being able to influence how the winning candidate chooses policy. ${ }^{2}$

The uncertainty about the vote count captures the idea that candidates cannot anticipate the outcome of the election, because they do not have enough information about the electorate. The candidates cannot anticipate whose vote will count and whose will not, so even though they anticipate the support intentions of the whole electorate, they are still uncertain about the electoral outcome.

The strategy of each citizen $i$ has two components: An entry strategy, determining whether to run as a candidate or not, and a support strategy, which determines for any possible set of candidates which one will citizen $i$ support.

Let $I_{i} \in\{0,1\}$ denote citizen $i$ 's entry strategy, where $I_{i}=1$ denotes entry and let $I=\left(I_{1}, \ldots, I_{N}\right)$ be the entry strategy profile of all the citizens. The set of candidates resulting from the entry strategy $I$ is $C \subseteq \mathcal{N}$. Let $\sigma_{i}: 2^{N} \longrightarrow \mathcal{N}$ denote the support strategy used by citizen $i$. Let $\sigma=\left(\sigma_{1}, \ldots, \sigma_{N}\right)$ be the support

\footnotetext{
${ }^{2}$ See Lee, Moretti and Butler (2004).
} 
strategy profile of every citizen in the society and let $\sigma_{-i}$ be the strategy profile of every citizen other than $i$.

The equilibrium concept we use is Undominated Subgame Perfect Nash Equilibrium in pure strategies, ruling out weakly dominated strategies. An equilibrium is defined by an entry strategy profile $I^{*}$ and a support strategy profile $\sigma^{*}$ such that:

(i) Given any set of candidates $C \subseteq \mathcal{N}, \sigma^{*}(C)$ is an Undominated Pure Nash equilibrium at the support stage. ${ }^{3}$

(ii) Given $\sigma^{*}$, the entry strategy profile $I^{*}$ is a pure Nash equilibrium at the entry decision stage of the game.

We let $C^{*}$ denote the set of candidates who run in the equilibrium $\left\{I^{*}, \sigma^{*}\right\}$.

We assume that each agent $i$ has a unique favorite policy $p_{i}=\underset{p \in[0,1]}{\arg \max } v_{i}(p)$ and we label and order individuals according to their favorite policy, so that for all $i, j \in \mathcal{N}, i<j$ implies $p_{i} \leq p_{j}$. Given this ordering of citizens, we assume that the preference profile satisfies the Strict Single-Crossing property:

Definition 1 A preference profile satisfies the Strict Single-Crossing property if for all $x, y \in[0,1]$ and all $i, j \in \mathcal{N}$ such that $y>x$ and $j>i, v_{i}(y) \geq v_{i}(x) \Longrightarrow$ $v_{j}(y)>v_{j}(x)$.

This property implies that if a "left-leaning" citizen $l$ prefers the right-most of two policies, then every citizen who is more right-leaning than $l$ also prefers the right-most policy. Given two policies, one more liberal, one more conservative, there cannot be any overlapping so that "conservative" citizens support the liberal policy and some more "liberal" citizens support the conservative policy: With strictly single-crossing preferences, given any two policy positions $p^{\prime}<p^{\prime \prime}$, there exists a cut-off point $\widehat{p}\left(p^{\prime}, p^{\prime \prime}\right)$ such that every citizen with an ideal policy below $\widehat{p}\left(p^{\prime}, p^{\prime \prime}\right)$ prefers $p^{\prime}$ to $p^{\prime \prime}$ and every citizen with an ideal policy above $\widehat{p}\left(p^{\prime}, p^{\prime \prime}\right)$ prefers $p^{\prime \prime}$.

Borrowing the terminology of the Poole and Rosenthal (1985) Nominate Scores model, single-crossing preferences imply "perfect voting": The voting behavior of a citizen-voter faced with a binary choice should be perfectly explained by the position of the citizen in the unidimensional "low-to-high" scale. Poole (2005) notes that in practice, more than $90 \%$ of the votes in US Congress can be explained by the position of a legislator in a one-dimensional policy space. $^{4}$

We introduce the following notation:

Let $m$ denote the median voter if $N$ is odd, and let $m l$ and $m h$ denote the two medians if $N$ is even. Labelling citizens by the relative position of their ideal policy from lowest to highest, $m$ is the citizen in position $\frac{N+1}{2}$ if such fraction

\footnotetext{
${ }^{3}$ With positive uncertainty, $\mu>0$, any weakly dominated support strategy is also strictly dominated in the support subgame and the set of Undominated Nash Equilibria coincides with the set of Nash Equilibria.

${ }^{4}$ This is merely suggestive, since we have in mind elections with electorates of any size and Poole refers to votes in the US Congress, whose size from 1789 on has never exceeded 525.
} 
is an integer; otherwise $m l$ and $m h$ are respectively the citizens in positions $\frac{N}{2}$ and $\frac{N+2}{2}$.

Let $S_{i}(C, \sigma)$ denote the support for candidate $i$, that is, the number of citizens whose support strategy is to support candidate $i$, given that the set of candidates is $C$ and the joint strategy profile is $\sigma$.

Let $V_{i}$ denote the number of valid votes for $i$. Therefore, the difference $S_{i}-V_{i}$ corresponds to the number of citizens who support $i$ but are unable to cast a valid vote for $i$ and can be interpreted as the number of "lost votes" for $i$.

In any race with two candidates $C=\{i, j\}$ with ideal policies $p_{i}, p_{j}$, let $\widehat{p}_{i j}$ denote the cut-off point such that every citizen with an ideal policy less than $\widehat{p}_{i j}$ prefers the candidate with a lower ideal policy, every citizen with an ideal policy above $\widehat{p}_{i j}$ prefers the candidate with the higher ideal policy, and only citizens with an ideal policy equal to $\widehat{p}_{i j}$ are indifferent between $i$ and $j$.

Note that if $p_{m}<\widehat{p}_{i j}$, the median prefers the candidate with the low ideal policy; say it is candidate $i$. Then $i$ will have more support than $j$ and $i$ would win the election if there were no uncertainty about the vote totals given the level of support. If no votes were lost, the margin of victory for $i$ would be $S_{i}-S_{j}$. In the actual outcome of the election, if $i$ loses more votes than $j$, there is a "shift" in the results that favors candidate $j$ and the margin of victory for $i$ is only $V_{i}-V_{j}$. If the difference in lost votes is big enough to offset the difference in initial support, $j$ wins the election. ${ }^{5}$

In any two-candidate race with $C=\{i, j\}$, let

$$
X_{i j}=\left(S_{i}-S_{j}\right)-\left(V_{i}-V_{j}\right)=\left(S_{i}-V_{i}\right)-\left(S_{j}-V_{j}\right)
$$

denote the "shift" from candidate $i$ to candidate $j$ in the difference of voting totals for the two candidates, compared to the original difference in support for the two candidates prior to the distortion introduced by the loss of votes. In short, $X_{i j}$ is equal to the number votes lost by $i$ minus the number of votes lost by $j$.

$X_{i j}$ is a discrete random variable: Let $f_{i j}(x)$ be its probability mass function and let $F_{i j}(x)$ be its distribution function, so $f_{i j}(x)$ is the probability that $X_{i j}=x$ and $F_{i j}(x)=\sum_{k=0}^{x} f_{i j}(k) .^{6}$

Note that in a race with two candidates $i$ and $j$, in which $i$ has $x$ more supporters, the probability that $i$ wins the election is

$$
F_{i j}(x-1)+\frac{1}{2} f_{i j}(x) .
$$

We are now ready to present our results.

\footnotetext{
${ }^{5}$ We can interpret our model as a simplified probabilistic voting model, in which we don't need exogenous non-policy preferences on the part of the voters to generate uncertainty about the electoral outcome.

${ }^{6}$ Let $b i[n, q ; k]$ be the probability that a binomial $b i[n, q]$ takes a value of $k$. Then:

$$
f_{i j}(x)=\sum_{k=0}^{N-1} b i\left[S_{i}, \mu ; k+x\right] b i\left[S_{j}, \mu ; k\right] .
$$
}




\section{Equilibria}

Let us first present the benchmark case in which there no uncertainty about the vote count $(\mu=0)$ and the candidates can anticipate the outcome of the election. ${ }^{7}$

A single candidate equilibrium exists if and only if $N$ is odd, whereas two candidate equilibria generically do not exist if $N$ is odd and they exist if $N$ is even. $^{8}$ Equilibria with multiple candidates are also possible.

These differences depending on the exact number of citizens are plausible in an election with a small electorate, such as a vote in a committee. However, in any election with a very large electorate, there will be some uncertainty about the number of votes each candidate will get and the results of the model should not depend on whether the size of the electorate is odd or even.

In the rest of the paper, we capture the uncertainty assuming that $\mu>0$ and we show that if the electorate is sufficiently large, whether $N$ is odd or even is irrelevant for the existence of equilibria with one or two candidates in elections with a large reward for holding office.

\subsection{Single Candidate Equilibrium}

In a unidimensional space, a unique median is a Condorcet winner and will have more support (and more expected vote share) than the other competitor in any two-candidate race. Nevertheless, the uncertainty about the vote totals gives any other candidate challenging the median some positive probability of winning the election.

Suppose that citizen $m+1$, the next citizen after the median, runs against the median $m$ in a two-candidate race. Then the median and everyone to her left support $m$ and the rest of the society supports $m+1$. As a result, candidate $m+1$ trails the median by one in terms of support.

If the median $m$ loses one more vote than $m+1$, the shift $X_{m, m+1}$ in favor of $m+1$ equals one and the election is tied. If $X_{m, m+1}$ is bigger than one, then $m+1$ wins the election.

Similarly, for any citizen $h>m$ running against the median, every citizen with an ideal policy above the cutting point $\widehat{p}_{m, h}$ will support $j$, and the rest will support the median. Suppose this means the median has $k$ more supporters. Then $j$ would need a shift in his favor of $k$ to tie the election and an even larger shift to win outright.

If the probability that there is such a big enough shift in favor of the weaker candidate is sufficiently high given the incentives to run (benefits of holding office and choosing policy), then a weaker candidate will be willing to run against the median.

We obtain the following result:

\footnotetext{
${ }^{7}$ This benchmark corresponds to the Besley and Coate (1997) model with the additional assumptions of strict single-crossing preferences in a unidimensional policy space, and $b>2 c$.

${ }^{8}$ This is mentioned in Eguia (2003).
} 
Lemma 1 There exists a single candidate equilibrium if and only if $N$ is odd, the median is unique, and for any citizen $j \in \mathcal{N} \backslash m$,

$$
\left(b+v_{j}\left(p_{j}\right)-v_{j}\left(p_{m}\right)\right) \operatorname{Pr}\left[W_{j}=1 \mid C=\{m, j\}\right] \leq c .
$$

In this equilibrium the median runs unopposed. ${ }^{9}$

This and all other proofs are shown in the Appendix.

Now note that as the electorate gets larger, the number of lost votes will increase. A candidate trailing by a small number of supporters will almost certainly lose if the electorate is also small, but a candidate trailing by the same small number of supporters will have a better chance of victory if the electorate is large and the difference in support is slim relative to the number of votes that can be lost. For instance, in an electorate with 5 citizens, a 3-2 split of support will give the weaker candidate a very small chance of victory. However, in an electorate with millions of citizens, a split of support in which the stronger candidate has only one more supporter is a virtual tie, and both candidates have an almost equal probability of victory.

In fact, for any fixed finite difference in support, if $\mu>0$ the probability of victory converges to a half for both candidates as the electorate gets larger. We use this result for our first theorem on existence of single candidate equilibrium in large societies.

Theorem 1 If the electorate is sufficiently large, then there is no single candidate equilibrium.

Suppose the benefit of holding office is three times the cost of running. Then any candidate with a one-third chance of victory will be willing to run. The probability of victory for a weaker candidate with one less supporter than the median is more than a third if $N \geq 103$ for $\mu=0.05$; or if $N \geq 1087$ for $\mu=0.005$. Since void ballots exceed $0.5 \%$ in most elections, these numerical examples show that the theorem applies for relatively small electorates. A higher degree of uncertainty about counting an individual vote has the same effect as increasing the size of the population: In either case it becomes harder for the agents to anticipate the outcome of the election and a challenger will have greater incentives to run against the median.

In an election with a small electorate the median can run unopposed because any other candidate would have only a very slim chance of beating the median. The median must be unique to run unopposed, so it is crucial that the electorate is odd. However, as the electorate gets larger, the uncertainty about the vote count gives other candidates challenging the median a better chance of winning, and for a sufficiently large society the probability of victory for citizens with an ideal point close to the median is high enough so that they will run and not let the median win unopposed, not even a unique, Condorcet winner median.

\footnotetext{
${ }^{9}$ The probability $\operatorname{Pr}\left[W_{j}=1 \mid C=\{m, j\}\right]$ is:

(i) $\frac{1}{2} f_{m j}(2 k+1)+1-F_{m j}(2 k+1)$ if $\widehat{p}_{m, j} \in\left(p_{m-k-1}, p_{m-k}\right) \cup\left(p_{m+k}, p_{m+k+1}\right)$

or (ii) $\frac{1}{2} f_{m j}(2 k)+1-F_{m j}(2 k)$ if $\widehat{p}_{m, j} \in\left\{p_{m-k}, p_{m+k}\right\}$, for a positive integer $k$.
} 


\section{$3.2 \quad$ Two-candidate equilibria}

Two candidates are a common feature of plurality elections, yet they are generically non-existent in a model without uncertainty if the number of citizens is finite and odd. We show that introducing uncertainty in the model guarantees the existence of two-candidate equilibria in every society with a large electorate.

Without uncertainty, a joint support strategy profile such that an entering third candidate receives no support from any other citizen is usually part of a two-candidate equilibrium: No third candidate enters because no one would support the entrant, and no one supports the entrant because since no one else does, supporting the doomed entrant is a waste.

With uncertainty, an entrant with no supporters still has a positive probability of victory. Suppose $i$ is an arbitrary citizen who sincerely prefers the entrant. If citizen $i$ is indifferent or almost indifferent between the two top candidates, then $i$ might want to deviate from the proposed equilibrium support strategy and support the entrant. Citizen $i$ then forfeits the chance of tilting the election in favor of one of the top two candidates. As the size of the electorate increases, the probability that the entrant with a single supporter wins given that a single vote decides the election converges towards zero; if the electorate is large and $i$ still supports the entrant, $i$ is wasting his support on a candidate who is asymptotically hopeless even on narrow elections decided by a single vote. ${ }^{10}$

We introduce an assumption ruling out the sort of preferences according to which some citizen is willing to be the only one supporting a virtually hopeless candidate: given any three candidates such that two of them receive enough support to be willing to run against each other, no citizen wants to be the sole supporter of the third candidate. Informally, each citizen thinks: "If no one supports the weak candidate, neither will I." We formalize this idea as a "No Lone Supporter" Assumption:

Assumption 1 (No Lone Supporter) Given an arbitrary support strategy $\sigma$, let $\{l, r\}$ be any pair of citizens willing to run a two-candidate race against each other and let $h, i \in \mathcal{N}$. Suppose that given $\sigma$, if the set of candidates is $C=\{l, r, h\}$, no citizen in $\mathcal{N} \backslash\{h, i\}$ supports $h$. Then, given $C=\{l, r, h\}$ and $\sigma_{-i}$, to support $h$ is not a best response for $i$.

No Lone Supporter is a joint assumption on preferences and the size of the electorate, becoming less restrictive as the size of the electorate increases. With preferences satisfying No Lone Supporter, for any two candidates willing to run against each other, there exists an equilibrium support strategy by all citizens in which no citizen would support an entrant. We only need to add the restriction that no citizen is willing to become a candidate if no other citizen would support her. We refer to this assumption as "Minimal Support".

Assumption 2 (Minimal Support) Given any entry and support strategy profile of all citizens other than $i$ such that none of them would support $i$ if $i$ run as a

\footnotetext{
${ }^{10}$ Citizen $i$ only takes into account outcomes decided by a single vote when considering which candidate to support, because in any other outcome $i^{\prime} s$ support is irrelevant.
} 
candidate, citizen $i$ prefers not to run. Formally, $\forall i \in \mathcal{N}$, given any $\left\{I_{-i}, \sigma_{-i}\right\}$ s.t. $S_{i}\left(I_{-i}, I_{i}^{\prime}, \sigma_{-i}, \sigma_{i}^{\prime}\right) \leq 1$ for any $\left\{I_{i}^{\prime} \sigma_{i}^{\prime}\right\}, I_{i}^{*}=0$ in any best response $\left\{I_{i}^{*}, \sigma_{i}^{*}\right\}$ of $i$ to $\left\{I_{-i}, \sigma_{-i}\right\}$.

For equilibria with two candidates, Minimal Support places a very mild restriction on the reward $b$, the cost $c$ and the preference profile to avoid entry by an unsupported third candidate. Since a third candidate with no supporters is not drawing support away from other candidates, she only affects the outcome of the election if she wins, and with no supporters the probability that she wins is virtually zero. Minimal Support merely requires that given such a negligible probability of obtaining a benefit from running for office, a candidate with no supporters would rather not participate in the election and not pay the cost of running. With multiple candidates, Minimal Support has stronger implications, as we shall discuss in the next subsection.

With these two assumptions we can focus our attention on finding two candidates $i$ and $j$ who would want to run against each other for office. Then we can construct an equilibrium with these two candidates in which no citizen would support an entrant and no third citizen will enter the race.

Theorem 2 Suppose the No Lone Supporter and the Minimal Support assumptions hold. Then if the electorate is sufficiently large, a two-candidate equilibrium exists.

The proof is constructive. If there is a unique median, in the absence of uncertainty (or with little uncertainty in small electorates), two-candidate equilibria do not exist unless the median citizen is indifferent between the two candidates, an event that generically does not occur. The probability that a stronger candidate with just one more supporter wins the election is very high, and trailing candidates will be discouraged at the long odds against them.

However, as the electorate grows, a positive uncertainty raises the probability that a candidate trailing by one vote will win. If there are large benefits of holding office, the same intuition that denied the existence of single candidate equilibria for large societies guarantees the existence of two-candidate equilibria for large electorates: In a large electorate, the probability that a weaker candidate trailing by a finite number of supporters will win the election is close to one half, and $b>2 c$ guarantees that such a weaker candidate will want to run, and that equilibria with two candidates exist in large societies, whether the number of citizens is even or odd.

Now that we have guaranteed existence for large electorates, we proceed to characterize the equilibria with two candidates $i$ and $j$, for the generic case in which no citizen is indifferent between two distinct ideal policies.

Assumption 3 (No Indifference) For any $i, j, l \in \mathcal{N}$ such that $p_{i} \neq p_{j}, v_{l}\left(p_{i}\right) \neq$ $v_{l}\left(p_{j}\right)$.

Without further loss of generality, assume $i<j$, so $p_{i} \leq p_{j}$. Let then $q(x)$ be the minimum number of supporters that $i$ must have in order for $i$ to win 
with probability no less than $x$, given that every other citizen will support $j$. That is, $q(x)$ is the minimum $k$ such that

$$
\operatorname{Pr}\left[W_{i}=1 \mid C=\{i, j\}, S_{i}=k, S_{j}=N-k\right] \geq x .
$$

Two candidates $i$ and $j$ can run in equilibrium if both have enough supporters to have a high enough probability of winning.

Proposition 2 Suppose the No Lone Supporter, Minimal Support and No Indifference assumptions hold. Then there exists a two-candidate equilibrium in which $i$ and $j$ run against each other if and only if:

$\widehat{p}_{i j} \in\left(p_{L}, p_{H}\right)$, where $L=q\left(\frac{c}{b+v_{i}\left(p_{i}\right)-v_{i}\left(p_{j}\right)}\right)$ and $H=q\left(1-\frac{c}{b+v_{j}\left(p_{j}\right)-v_{j}\left(p_{i}\right)}\right)$, $L, H \in \mathcal{N} .{ }^{11}$

There is a two-candidate equilibrium if both candidates get a similar number of supporters and thus they both have a sufficient probability of winning. In order for the electorate to split in roughly equal halves, the cutting point $\widehat{p}_{i j}$ between those who support $i$ and those who support $j$ must be close to the median. Proposition 2 shows that in particular, it has to be in between the ideal point of citizens $L$ and $H$, where the identity of $L$ and $H$ is specified using the function $q(x)$.

Since $i$ is the candidate with a lower ideal policy, in order for $i$ to run, it must be that (at least) the first $L$ citizens support $i$. But since $b>2 c$, candidate $i$ is willing to run for a probability of victory which is less than a half. It follows that citizen $L$ is a citizen weakly to the left of the median $m$ (if $N$ is odd) or of the low median $m l$ (if $N$ is even), for if the median or lower median support $i$, the probability of victory for $i$ is at least a half. Similarly, $H$ is a citizen weakly to the right of the median or the high median $m h$. Suppose $N$ is even. Then it follows $\widehat{p}_{m l, m h} \in\left(p_{m l}, p_{m h}\right) \subseteq\left(p_{L}, p_{H}\right)$, thus there exists a two-candidate equilibrium in which $m l$ and $m h$ run against each other.

Corollary 3 Under the No Lone Supporter and Minimal Support assumptions, if $N$ is even a two-candidate equilibrium exists.

If the reward for holding office is big, in a small society with an odd number of citizens, equilibria with two candidates typically do not exist. On the other hand, in a society with a large, or a small but even number of citizens, there always exists a two-candidate equilibrium.

We now address the question of convergence to the median: In our constructive proof of Theorem 2, the median(s) or a citizen very close to the median are the two candidates in equilibrium. However, this need not be the case: Two very extreme candidates $i$ and $j$ can run in equilibrium, insofar as the cutting point $\widehat{p}_{i j}$ is very close to the median, (or to the medians if $N$ is even).

In an equilibrium without uncertainty $(\mu=0)$, the two candidates must have equal support. With uncertainty, we only require that they have similar, not

\footnotetext{
${ }^{11}$ Without No Indifference, $\widehat{p}_{i j} \in\left(p_{L}, p_{H}\right)$ is a sufficient condition and $\widehat{p}_{i j} \in\left[p_{L}, p_{H}\right]$ a necessary one. We sketch the proof of this claim in the Appendix.
} 
necessarily equal support. In larger societies, the margin by which the weaker candidate trails in support may be bigger in absolute terms, but the fraction of the population that supports each candidate should converge to a half in a two-candidate equilibrium as the electorate gets larger. If the weaker candidate lags heavily in support, he should abandon the race.

The cutting point $\widehat{p}_{i j}$ will have to be close to the median in order for the candidates to have similar support. But the candidates themselves need not be close to the median. We do not have a convergence result in terms of the policy that will ultimately be implemented, but only in terms of the "undecided voter", the citizen who is indifferent between the two candidates. This citizen (if it exists) ought to be close to the median, splitting the electorate into two halves of roughly the same size, so that both candidacies are competitive.

The two candidates can be two moderates, or two extremists (one from each extreme), or anything in between so long as they split society into two similarly big groups. To address this multiplicity of equilibria, we select equilibria according to their robustness to the assumption on citizens' support behavior. We introduce a new concept of equilibrium, which allows for both strategic and sincere support behavior on the part of the agents.

Definition 2 A Sincere-Strategic Equilibrium is a pair $\left\{I^{*}, \sigma^{*}\right\}$ such that:

(i) The support strategy profile $\sigma^{*}$ is an Undominated Pure Nash Equilibrium of the support stage for any set of candidates $C \subseteq \mathcal{N}$.

(ii) The entry strategy profile $I^{*}$ is a Pure Nash Equilibrium at the entry stage given any subset $E \subseteq \mathcal{N}$ such that at the support stage every citizen $i \in E$ follows the strategy $\sigma_{i}^{*}$ and every citizen $j \notin E$ follows a sincere support strategy.

In a Sincere-Strategic equilibrium, the set of candidates $C^{*}$ must be the equilibrium outcome of the entry stage regardless of whether every citizen acts strategically or sincerely in the support stage. Furthermore, $C^{*}$ must also be the equilibrium outcome of the entry stage if any subset of agents deviate from the strategic equilibrium and choose whom to support sincerely.

Published estimates of the incidence of strategic voting behavior in the US and the UK (two countries that elect a single representative per district) range between $5 \%$ and 17\%, as reported by Alvarez and Nagler (2000). Our SincereStrategic equilibrium is a Strategic equilibrium which is robust at the entry stage to deviations towards sincerity by any coalition of agents. While it is difficult for citizens to coordinate strategically, it is easier to hypothesize how they might be convinced as a group to vote sincerely: An appeal by a candidate simply to the "honesty" of the citizens, to "vote according to your heart" might convince a large number of voters to deviate from the equilibrium strategic behavior more readily than a complicated appeal to coordinate on a sophisticated deviation. Our refined equilibrium concept requires the equilibrium to be robust to successful appeals to sincerity.

It is trivial to note that every single candidate equilibrium is a SincereStrategic equilibrium: In a single candidate equilibrium, citizens automatically elect the only candidate and if a second candidate entered the race, with two 
candidates strategic support is equivalent to sincere support. Therefore, whether citizens choose whom to support sincerely or strategically does not alter the entry decision of any citizen when there is a single candidate running.

However, if there are two candidates running, citizens who act sincerely may support an entrant who would have been shunned if all citizens acted strategically.

In particular, note that a two-candidate equilibrium with two extreme candidates cannot be a sincere-strategic equilibrium, because a moderate entrant could gather a significant amount of support, enough to have a high enough probability of victory. Thus some convergence is necessary for a sincere-strategic equilibrium.

Nevertheless, full convergence is not possible: Two adjacent candidates would also fail to stand in a two-candidate sincere-strategic equilibrium, because another adjacent candidate, just a little bit more extreme than either of the two original ones can enter and outflanking one of the candidates, get the support of almost a half of the electorate. For example, if $m l$ and $m h$ were running, then $m h+1$ can run, and under sincere supporting behavior, get the support of $\frac{N}{2}-1$ citizens, leaving $m l$ with $\frac{N}{2}$ supporters and $m h$ with just his own support.

Remark 4 Sincere-Strategic equilibria with two candidates will consist of a leftleaning candidate and a right-leaning candidate, satisfying three conditions:

(i) They are both separate from the median,

(ii) they are not too far from each other,

(iii) and they split society into two groups with roughly the same number of supporters.

The candidates have to be separate from the median to make an extreme third candidacy inviable: If a third candidate entered to the left of the leftleaning candidate, the right-leaning candidate would win if enough leftist citizens were sincere and the left vote is split, or else the entrant would have no support and would not affect the election (if citizens are strategic).

They cannot be too far from each other (too extreme) or else a moderate third candidate would enter in between them and the entrant would sweep the election if citizens chose support sincerely.

The two candidates have to split society into two roughly equal groups of support to be both viable candidates with a good chance of winning the election.

In the Appendix we show a set of sufficient assumptions that guarantee the existence of a Sincere Strategic equilibrium with two candidates in a large electorate. These assumptions include Euclidean preferences, two mild restrictions on the distribution of ideal policies, which we want to be somewhat evenly spread and not too clumped near the median, and a more restrictive assumption on the cost of running as a candidate, which we require to be high enough to deter entry by candidates with no chance of winning.

Here we just introduce an example: 
Example 1 Let there be 100 citizens, with ideal policies $p_{i}=\frac{i}{100}$ for all $i \in$ $\{1,2, \ldots 100\}$. Let the policy preferences be:

$$
v_{i}(p)=\left\{\begin{array}{l}
-0.1\left(p_{i}-p\right) \text { if } p \leq p_{i} . \\
-0.101\left(p-p_{i}\right) \text { if } p>p_{i} .
\end{array}\right\}
$$

so every citizen $i$ prefers the candidate who is closest to $i$ and in case of equal distance, the candidate with the lower ideal policy. Let uncertainty $\mu=0.025$ and let $b=10 c=10$.

Then, in any two candidate Sincere-Strategic equilibrium with $C=\{i, j\}$ :

Either $(i, j) \in\{(47,53),(48,53)\}$ or $i \in\{17,18, \ldots, 47\}, j \in\{54,55 \ldots 83\}$, $\frac{(i+j)}{2} \in[49,51.5]$, and $|i-j| \leq 64$.

We provide calculations in the Appendix. This example illustrates the result: If two candidates run against each other, one will be left-of-the-median, one will be right-of-the-median, they will not be too extreme, and there will be some gap between them.

\subsection{Equilibria with 3 or more candidates}

Equilibria with multiple candidates are possible in our model.

Models without uncertainty can distinguish between "winning candidates" (those who win with positive probability) and "spoilers" (those who run just to affect the outcome indirectly, but with no probability of actually winning). With uncertainty, every candidate has some positive probability of victory, albeit possibly a very small one, regardless of the support they have. Nevertheless, we can still describe some candidates as spoilers: Those who trail in support and run mostly to influence who wins when they lose.

Formally, we can define "competitive candidates" as those who have enough of an incentive to run based just on their probability of winning, without taking into account the effect that their candidacy would have on the electoral outcome if they lose. Competitiveness is defined relative to a specific joint strategy of all citizens at the support stage; thus a candidate can be competitive for some profile of support and not competitive given a different support profile.

Definition 3 Given a joint support strategy profile $\sigma$, a candidate $i \in C$ is competitive if

$$
\left\{b+v_{i}\left(p_{i}\right)-\sum_{k \in C \backslash i} v_{i}\left(p_{k}\right) \operatorname{Pr}\left[W_{k}=1 \mid C \backslash i\right]\right\} \operatorname{Pr}\left[W_{i}=1 \mid C\right]>c,
$$

and a spoiler otherwise.

Competitive candidates run to win. Spoiler candidates run motivated by the advantages that running for a defeat entails for them.

Remark 5 In order for a pair $\left\{I^{*}, \sigma^{*}\right\}$ to be a multiple-candidate equilibrium, three conditions must hold: 
(i) Every candidate $i \in C^{*}$ prefers the lottery over outcomes with the set of candidates $C^{*}$, better than the lottery over outcomes if $i$ drops out and the set of candidates is $C^{*} \backslash i .^{12}$

(ii) No other citizen wants to enter the race.

(iii) Every citizen $j \in \mathcal{N}$ supporting $i \in C^{*}$ prefers the lottery over outcomes given $C^{*}$ and $\sigma^{*}$ to the lottery that results if $j$ deviates and supports some other candidate.

We present an example of a Sincere-Strategic equilibrium with three candidates.

Example 2 Let there be 183 citizens with Euclidean preferences $v_{i}(p)=\left|p-p_{i}\right|$. Let $b=4 c=4$. Let $p_{31}=0.2 ; p_{61}=0.25 ; p_{62}=0.4 ; p_{m}=0.5 ; p_{122}=0.6$; $p_{123}=0.76 ; p_{153}=0.8$. Finally, let uncertainty be $\mu=0.01$.

Denote citizen 31 by $l$ and citizen 153 by $r$. Then there exists a SincereStrategic equilibrium in which $C^{*}=\{l, m, r\}$.

In this equilibrium, every citizen sincerely supports the candidate with the closest ideal policy. With Euclidean preferences and no uncertainty, under mild assumptions three candidate equilibria do not exist (Besley and Coate); in Example 2 if $\mu=0$, citizen 62 would prefer to change her support from the median to the leftist candidate, giving $l$ the victory in the election. However, with uncertainty $\mu=0.01$, if citizen 62 supports the left candidate, then the three candidates $\{l, m, r\}$ have a total support of 62,60 and 61 , respectively. With these support totals, $l$ wins the election with probability close to $81 \%$, but $r$ wins with probability above $18 \%$, whereas the median wins only with probability close to $1 \%$. Citizen 62 would rather let each of the three candidates have an equal opportunity of victory than to almost completely sacrifice the chance to win of his favorite candidate while not quite securing that his second-best candidate will win. ${ }^{13}$ Similar calculations show that citizens 61, 122 and 123 (the most tempted to support their second best) also prefer to sincerely support their most preferred candidate.

This example can easily be extended to a large electorate: Let us introduce $n$ new agents, a sixth of them with an ideal policy below $p_{l}$, another sixth with ideal policies between $p_{l}$ and $p_{61}$, another sixth with ideal policies between $p_{62}$ and $p_{m}$, another sixth with ideal policies between $p_{m}$ and $p_{122}$, another sixth with ideal policies between $p_{123}$ and $p_{r}$ and the last sixth with ideal policies above $p_{r}$. Then $C^{*}=\{l, m, r\}$ and sincere support by every citizen is still a Sincere-Strategic equilibrium.

\footnotetext{
${ }^{12}$ Formally: (ii) For all $i \in C$,$$
b \operatorname{Pr}\left[W_{i}=1 \mid C\right]+\sum_{j \in C}\left|v_{i}\left(p_{i}\right)-v_{i}\left(p_{j}\right)\right|\left(\operatorname{Pr}\left[W_{j}=1 \mid C \backslash i\right]-\operatorname{Pr}\left[W_{j}=1 \mid C\right]\right)>c .
$$

${ }^{13}$ If the three candidates stand an equal chance of victory, the utility for citizen 62 is $-\frac{0.2+0.1+0.4}{3}=-0.233$. If citizen 62 votes for $l$, the utility for citizen 62 is approximately $-0.2 * 0.805-0.1 * 0.014-0.4 * 0.181=-0.235$.
} 
Equilibria with four candidates may have one or two competitive candidates, as in Besley and Coate, or three or four, as in Osborne and Slivinski. However, if we consider the refinement of a Sincere-Strategic Equilibrium, then we find that spoilers must all be in between two competitive candidates. Therefore, equilibria with a single competitive candidate will not be Sincere-Strategic.

Lemma 6 In any Sincere-Strategic equilibrium with multiple candidates, if $i \in$ $C$ is a spoiler, then there exist $j, h \in C$ such that $p_{i} \in\left(p_{j}, p_{h}\right)$.

Corollary 7 In any Sincere-Strategic equilibrium with two or more candidates, there exist at least two competitive candidates.

If preferences are Euclidean we can show that if the electorate is sufficiently large, then all candidates will have a similar support. We consider a sequence of societies of increasing size $N$ and we show that the result holds for all societies larger than some size $n$. To prove this we need to add a technical assumption:

Assumption 4 There exists a set $Q \subset[0,1]$ composed of finitely many points such that for all $N$ and for all $i \in N, p_{i} \in Q$. Furthermore, for any $q, q^{\prime} \in Q$, $\frac{q+q^{\prime}}{2} \notin Q$.

Assumption 4 guarantees that the number of distinct ideal policies is bounded as the size of the electorate approaches infinity. The second part of the assumption holds generically: if the points in $Q$ are randomly chosen between zero and one, the probability that the midpoint between two of them is also chosen is zero.

With this extra assumption we can show that if agents have Euclidean preferences, then in any SS equilibrium with a large electorate all candidates receive an approximately equal share of support.

Proposition 8 Suppose that preferences are Euclidean and suppose that Minimal Support and Assumption 4 hold. Then, for any positive $\epsilon$ there exists a positive integer $n$ s.t. if $N>n$, in any Sincere Strategic equilibrium $\frac{S_{i}}{S_{j}}>1-\epsilon$ for all $i, j \in C^{*}$.

The intuition for the proof is as follows: If the electorate is large enough, a candidate whose share of support is less than that of the strongest candidates will lose with probability approaching one. Then, by Lemma 6 and Corollary 7 , it must be that this weak candidate is a spoiler, between two competitive candidates. But then, given two competitive candidates and a trailing weak candidate, votes for the spoiler are wasted votes, and only citizens who are indifferent among the top two candidates will support the spoiler. We have ruled out (non-generic) indifference between two candidates with distinct ideal policies by assumption and as a result the spoiler will get no votes. According to Minimal Support, citizens with no support don't run as candidates. Note that Minimal Support, rather mild in the case of equilibria with two candidates, now has stronger implications. In particular, Minimal Support rules out equilibria 
in which a candidate with zero support runs because if she dropped out, the offequilibrium path strategies of other citizens are such that the support to other candidates would change and thus, even with zero supporters, the candidate affects the outcome of the election. A high enough cost of running that deters entry by candidates with only a negligible chance of victory is enough to satisfy Minimal Support. Alternatively, we can interpret Minimal Support as a restriction on the class of strategy profiles that agents can play. It is difficult to justify why agents would change their support after a candidate that no one supports drops out. It is more plausible that the strategy profiles played in equilibria are such that the entry decision of a citizen who no one supports is irrelevant to the outcome of the election. If so, our Minimal Support assumption is satisfied.

A complete characterization of multi-candidate equilibria is beyond the scope of this paper.

\section{Conclusion and Extensions}

We have introduced a model of representative democracy with endogenous candidates and uncertainty about the total vote count. We predict that the median will be able to run a successful, unopposed campaign only if the number of citizens is small and odd. If the number of citizens is even or the electorate is large, no citizen can run unopposed and a two-candidate equilibrium will exist.

In a model with a finite electorate, strategic voting, no uncertainty and a large benefit from holding office, two-candidate equilibria generically do not exist if the number of citizens is odd, whereas they exist if $N$ is even. Introducing uncertainty, we match these results for a small electorate, but in a large electorate we find that the exact number of citizens is irrelevant and there always exist a two-candidate equilibrium if the benefit from holding office is at least twice as high as the cost of running.

We have introduced a refined equilibrium concept, Sincere-Strategic Equilibrium, which requires the equilibrium set of candidates to hold regardless of whether citizens choose which candidate to support strategically or sincerely, and requires the equilibrium of the entry stage to be robust to coalitional deviations from strategic to sincere decision-making rules. We show that if there exists a Sincere-Strategic equilibrium with two candidates, the candidates cannot be too extreme, but one will lean to the left and the other one to the right; thus, policies do not converge to the median. We show a set of sufficient conditions for this equilibrium to exist.

With certain restrictions on the strategies used by the citizens, the refined equilibrium concept rules out all multi-candidate equilibria in which some candidates run with little support. If equilibria with three or more candidates exist, then all candidates must have a similar share of support.

We now compare our results with those of the two most prominent models of representative democracy with endogenous candidates: Osborne and Slivinski's (1996) and Besley and Coate's (1997).

Besley and Coate consider a finite number of strategic citizens. Candidates 
perfectly anticipate the outcome of the election, which depends crucially on the exact (odd or even) number of citizens: Assuming that the benefit of holding office is large, single candidate equilibria exist if the median is unique ${ }^{14}$ and two candidate equilibria exist if the number of citizens is even. This model best fits an election with a very small electorate. With such an electorate, our model yields similar results: Single candidate equilibria exist if the median is unique and two-candidate equilibria exist if the number of citizens is even.

It is reasonable that the exact number of citizens determines the equilibrium outcome in a society with a very small electorate, but it is a less plausible prediction in a society with a large electorate. We have shown that the uncertainty in our model makes the exact number of citizens irrelevant in large societies: Equilibria with a single candidate will not exist, and equilibria with two candidates will exist. The key difference between our model and Besley and Coate's is that under uncertainty about the vote count, a candidate trailing by a small margin in terms of expected votes will have a high enough probability of victory if the electorate is large enough and therefore this candidate will choose to run. If the election was deterministic, no citizen could successfully challenge the median.

Our result for large electorate reinforces the findings of Osborne and Slivinski, who reach a similar prediction under a number of simplifying assumptions (sincere voting, a continuum of voters, Euclidean preferences). Their model is bound to fit large electorates better than small ones, for it relies on the existence of an infinite number of voters.

Our model with a finite number of voters thus builds a bridge between the two main models of representative democracy with endogenous candidates, agreeing with each of the two models where it is most appropriate -with Besley and Coate for small electorates, with Osborne and Slivinski for large ones. We blend their different predictions into a single, unifying framework that captures the insights of large electorates where complete information is implausible as well as those of smaller electorates where the outcome is easier for all agents to foresee.

For future research, we would like to introduce not just endogenous candidates, but endogenous parties into the model: Some citizens choose to join a party, and the party chooses to field a candidate. Parties could be just coordination devices between citizens, or they could be cost-sharing devices that help to fund the campaign of the candidate they field.

\section{Appendix}

\subsection{Lemma 1}

Proof. If $N$ is even, both $m l$ and $m h$ will have at least a $50 \%$ chance of victory against any other citizen in a two-candidate race. Thus $b>2 c$ guarantees that they will both be willing to run against any single candidate, thus no citizen

\footnotetext{
${ }^{14} \mathrm{Or}$ if there exist a Condorcet winner if the policy space is not unidimensional.
} 
may stand unopposed. The same argument applies to all the medians if $N$ is odd and the median is not unique.

If $N$ is odd and the median is unique, the median would run against any other citizen who was standing alone, so no citizen other than the median can run unopposed.

If $N$ is odd and the unique median $m$ was running, then any other citizen $j \in \mathcal{N}$ would run against the median if and only if

$$
\left(b+v_{j}\left(p_{j}\right)-v_{j}\left(p_{m}\right)\right) \operatorname{Pr}\left[W_{j}=1 \mid C=\{m, j\}\right]>c .
$$

For every $j \in \mathcal{N}$ such that if $j$ and $m$ run, the cutting point in support is in $\left(p_{m-k-1}, p_{m-k}\right) \cup\left(p_{m+k}, p_{m+k+1}\right)$, the excess support of the median is $S_{m}-S_{j}=$ $2 k+1$, thus the probability of victory for $j$ is

$$
\frac{1}{2} f_{m j}(2 k+1)+\left(1-F_{m j}(2 k+1)\right) .
$$

For any $j \in \mathcal{N}$ such that if $j$ and $m$ run the cutting point in support is $p_{m-k}$ or $p_{m+k}$ for some $k$, the excess support of the median is $S_{m}-S_{j}=2 k$, thus the probability of victory for $j$ is $\frac{1}{2} f_{m j}(2 k)+\left(1-F_{m j}(2 k)\right)$.

\subsection{Theorem 1}

Proof. The only single candidate equilibrium is that in which a unique median is running (Lemma 1). Now suppose the next citizen, $m+1$, should challenge the median. Then the difference in support is 1 and the probability that the weaker candidate $m+1$ wins is $\frac{1}{2} f_{m, m+1}(1)+1-F_{m, m+1}(1)$, which is equal to

$$
1-\frac{1}{2} f_{m, m+1}(1)-f_{m, m+1}(0)-F_{m, m+1}(-1) .
$$

As $N$ tends to infinity, $f_{i, j}(x)$ converges to zero for any given integer $x$, in particular for $f_{m, m+1}(1)$ and $f_{m, m+1}(0)$, so the probability of victory for candidate $m+1$ and the expression $\left(1-F_{m, m+1}(-1)\right)$ converge to the same value. Since the median has more support, the probability that the median loses less votes than the other candidate is less than a half, so $F_{m, m+1}(-1)<\frac{1}{2}$ for all $N$, and $\left(1-F_{m, m+1}(-1)\right)>\frac{1}{2}$ for all $N$. Since the probability that $m+1$ wins is less than one half for all $N$, but it converges to the same value as $\left(1-F_{m, m+1}(-1)\right)$, it must be that both converge to $\frac{1}{2}$. Then given $b>2 c$, citizen $m+1$ will run against the median if the electorate is big enough.

\subsection{Theorem 2}

Proof. If $N$ is odd and $m$ and $m+1$ run in a two candidate race, the probability of winning converges to a half for each candidate as the number of citizens increases. Given $b>2 c$, both candidates want to run if the probability of victory is close enough to one half. 
If instead $N$ is even, then $m l$ and $m h$ can run against each other in equilibrium regardless of the size of the society, for they each have a one half probability of victory.

Entry by a third candidate is deterred by the No Lone Supporter assumption, which guarantees that there exists an equilibrium support strategy such that no citizen would support the entrant, and the Minimal Support assumption, which guarantees that no citizen would want to enter the race if she was not going to get a single supporter.

\subsection{Proposition 2}

Proof. By the No Lone Supporter assumption, if $N \geq 6$, no two citizens can have the same ideal policy. Suppose three citizens $l, l^{\prime}, l^{\prime}$ shared a common ideal policy. Then if $C=\left\{l, l^{\prime}, l^{\prime \prime}\right\}$, it would be a best response for any fourth citizen to support any of the three regardless of the support strategies by all other citizens, which contradicts No Lone Supporter. Suppose there are just two citizens $l, l^{\prime}$ with a common ideal policy and suppose $N \geq 6$, then there exist two citizens $k, k^{\prime}$ either such that $p_{k} \leq p_{k^{\prime}}<p_{l}$, or such that $p_{k} \geq p_{k^{\prime}}>p_{l}$. In either case if $C=\left\{l, l^{\prime}, k^{\prime}\right\}$, the best response for $k$ is to support $k^{\prime}$ regardless of the strategies of the other citizens, again in contradiction of No Lone Supporter. Therefore, if No Lone Supporter holds and $N \geq 6$, for any $i, j \in \mathcal{N}$ such that $i<j$, it must be $p_{i}<p_{j}$.

Thus, if $\widehat{p}_{i j}>p_{L}$, then $L$ and every other citizen $l$ with $p_{l} \leq p_{L}$ support $i$ and $i$ has at least $L$ supporters. Since $L=q\left(\frac{c}{b+v_{i}\left(p_{i}\right)-v_{i}\left(p_{j}\right)}\right)$, the probability that $i$ wins is

$$
\operatorname{Pr}\left[W_{i}=1 \mid C=\{i, j\}\right] \geq \frac{c}{b+v_{i}\left(p_{i}\right)-v_{i}\left(p_{j}\right)} .
$$

Citizen $i$ chooses to run if the expected benefit of running offsets the cost of entry. The expected benefit of running is:

$\left[b+v_{i}\left(p_{i}\right)-v_{i}\left(p_{j}\right)\right] \operatorname{Pr}\left[W_{i}=1 \mid C=\{i, j\}\right] \geq\left[b+v_{i}\left(p_{i}\right)-v_{i}\left(p_{j}\right)\right] \frac{c}{b+v_{i}\left(p_{i}\right)-v_{i}\left(p_{j}\right)}=c$

Thus, citizen $i$ wants to run.

Similarly, if $\widehat{p}_{i j}<p_{H}$, then $H$ and every other citizen $h$ with $p_{h} \geq p_{H}$ support $j$ and consequently $i$ has less than $H$ supporters. Thus, the probability that $i$ wins is less than $1-\frac{c}{b+v_{j}\left(p_{j}\right)-v_{j}\left(p_{i}\right)}$, or equivalently, the probability that $j$ wins is more than $\frac{c}{b+v_{j}\left(p_{j}\right)-v_{j}\left(p_{i}\right)}$. Therefore, $j$ also wants to run:

$\left[b+v_{j}\left(p_{j}\right)-v_{j}\left(p_{i}\right)\right] \operatorname{Pr}\left[W_{j}=1 \mid C=\{i, j\}\right]>\left[b+v_{j}\left(p_{j}\right)-v_{j}\left(p_{i}\right)\right] \frac{c}{b+v_{j}\left(p_{j}\right)-v_{j}\left(p_{i}\right)}=c$.

Under the No Lone Supporter assumption, the following support strategy is an equilibrium of the support stage: For any configuration $C$ of candidates such that $\{i, j\} \in C$, every citizen supports the candidate in $\{i, j\}$ she prefers most, and no citizen supports any other candidate. 
With this support strategy, an entrant would always receive zero votes from the rest of the electorate. The Minimal Support assumption is then sufficient to guarantee that no third candidate will join the race between $i$ and $j$.

Therefore, if $\widehat{p}_{i j} \in\left(p_{L}, p_{H}\right)$ there exists an equilibrium in which $i$ and $j$ run, and the rest of citizens would never support a third entrant.

If $\widehat{p}_{i j}<p_{L}$, then:

$\left[b+v_{i}\left(p_{i}\right)-v_{i}\left(p_{j}\right)\right] \operatorname{Pr}\left[W_{i}=1 \mid C=\{i, j\}\right]<\left[b+v_{i}\left(p_{i}\right)-v_{i}\left(p_{j}\right)\right] \frac{c}{b+v_{i}\left(p_{i}\right)-v_{i}\left(p_{j}\right)}=c$

and $i$ would not want to run against $j$; if $\widehat{p}_{i j}>p_{H}$, then $j$ would not want to run against $i$ :

$\left[b+v_{j}\left(p_{j}\right)-v_{j}\left(p_{i}\right)\right] \operatorname{Pr}\left[W_{j}=1 \mid C=\{i, j\}\right] \leq\left[b+v_{j}\left(p_{j}\right)-v_{j}\left(p_{i}\right)\right] \frac{c}{b+v_{j}\left(p_{j}\right)-v_{j}\left(p_{i}\right)}=c$.

15

Without No Indifference, $\widehat{p}_{i j}=p_{L}$ is also possible. Since no two citizens share the same ideal policy, if $\widehat{p}_{i j}=p_{L}$, then $L-1$ citizens support $i, L$ abstains and $N-L$ support $j$. From the construction of $L$ we know that if $L$ citizens support $i$ and $N-L$ support $j, i$ is willing to run, but if $L-1$ support $i$ and $N-L+1$ support $j$, then $i$ prefers to drop out of the race. Therefore, $\widehat{p}_{i j} \geq p_{L}$ is necessary and $\widehat{p}_{i j}>p_{L}$ sufficient for $i$ to be willing to run.

\subsection{Example 1}

Citizens will want to run if their chance of victory is better than $10 \%$ just out of motivation to hold office, and depending on who else is running, they may want to run for a chance of victory slightly less than $10 \%$, out of policy considerations. No citizen wishes to run for a probability of victory of less than $9 \%$.

If the two top candidates split support 49-51 or 48-50, the weaker candidate has more than $10.5 \%$ probability of victory, thus he is willing to run, but with a split of 48-51 or worse (trailing by three or more supporters), the weaker candidate has a probability of victory below $5 \%$ and would not want to run. With just two candidates $i$ and $j$, where we let $i$ be the lower candidate, citizens split support 49-51 if $i+j$ adds up to 98, 99 (in which case $i$ gets support from citizens 1 to 49), 102 and 103 (in which case citizens 50 and 51 support $i$ as well). Citizens split support 50-50 if $i+j$ adds up to 100 or 101 .

Thus in order for $i$ and $j$ to be willing to run against each other, it must be $98 \leq i+j \leq 103$.

However, if $j$ is 50 , or 51 , then a third candidate $h=52$ could enter and get 49 supporters (if citizens happen to be sincere), enough to make $h$ competitive. If $j=52$ and $i=49,50$ or 51 , then $l=48$ could enter and get at least 48

\footnotetext{
${ }^{15}$ To be rigorous, we need to add that if $\widehat{p}_{i j} \in\left(p_{H}, p_{H+1}\right)$, it could be that $\left[b+v_{j}\left(p_{j}\right)-\right.$ $\left.v_{j}\left(p_{i}\right)\right] \operatorname{Pr}\left[W_{j}=1 \mid C=\{i, j\}\right]=c$. If so, citizen $j$ has no incentives to run, but being indifferent, $j$ could decide to run against $i$. As this is an uninteresting knife-edge case, we have omitted it from the statements of Proposition 2 and Footnote 11.
} 
supporters under sincere support, leaving $i$ with 1 or 2 , and $j$ with no more than 50. Then $l$ would enter. If $j=52$ and $i<49$, then $h=53$ could enter and get 48 supporters, leaving $i$ with no more than 50 and $j$ with 2 or 3 . Then $h$ would run.

Therefore, it cannot be that $j$ is less than 53. And if $j=53$, then $i$ cannot be less than 47, or else $h=54$ with 47 supporters would enter and face $i$ with no more than 49 ( $j$ would keep the support of citizens 50, 51 and 52).

Similarly, if $i$ is 49 or 50 , then $l=48$ could enter and get 48 votes, enough to be competitive against the (no more than 50) votes of $j$.

If $i=48$ and $j=53$, then outflanking is no longer viable for a third candidate: If $l=47$ enters, then $l$ gets 47 votes and $j$ gets 50 .

So besides adding up to $98,99,100,101,102$ or 103 , it must be that either $i, j \notin\{48,49,50,51,52,53\}$ or $(i, j) \in\{(47,53),(48,53)\}$.

Finally, $i$ and $j$ cannot be too far apart, or else a moderate citizen will enter:

If $j-i$ is more than 64 , then either $m l=50$ or $m h=51$ could enter and get no less than 33 supporters and leave the other two candidates with 33 and 34 supporters (or 34 and 33). In such a tight three-way race, the probability that the weakest candidate wins the election is over $10 \%$.

\subsection{Sufficient conditions for existence of two-candidate SS Equilibria:}

Let $\eta=\min _{\{x\}} x$ s.t. $\frac{b}{2} f_{i j}(x)+b F_{i j}(x-1)>c$. That is, $\eta$ is the maximum amount of support by which a candidate $i$ could be trailing in a two candidate race and still be motivated to run solely for the expected benefit of holding office.

Let $I$ be the smallest convex interval containing the ideal policies of at least $\eta$ citizens.

Assumption 5 Any convex interval $I^{\prime} \subseteq[0,1]$ weakly larger than $I$ contains the ideal policy of at least 1 agent.

Let $\lfloor x\rfloor$ denote the largest integer smaller or equal to $x$ and let $\lceil x\rceil$ denote the smallest integer equal or larger than $x$. We use this notation in the following condition, which guarantees that the distribution of ideal policies is not too lopsided near the median.

Assumption 6 There exist a rational number $\varphi>0$ such that for all $N, p_{m}$ is closer to $p_{\lfloor m-\varphi N\rfloor}$ than to $p_{\lfloor N-3 \varphi N\rfloor}$ and also $p_{m}$ is closer to $p_{\lceil m+\varphi N\rceil}$ than to $p_{\lceil 3 \varphi N\rceil}$.

The interpretation of this condition is that cannot be that the ideal policies of almost a half of the population lie within some distance to the left of the ideal policy of the median, whereas within the same distance to right of the median lie very few ideal policies, nor can it be that there are a lot of ideal policies just to the right of the median and very few just to the left.

Let $c^{*}=\max _{i, j, l \in \mathcal{N}}\left|v_{i}\left(p_{j}\right)-v_{i}\left(p_{l}\right)\right|$ for all $i, j, l$. 
Euclidean preferences are defined by the Euclidean distance between the ideal policy of the agent and the implemented policy: $v_{i}(p)=\left|p-p_{i}\right|$.

Claim 9 Suppose preferences are Euclidean, Assumptions 5 and 6 hold and $c>$ $c^{*}$. There exist some $n$ such that if $N>n$, then a Sincere Strategic Equilibrium with two candidates exists.

Proof. For notational simplicity, suppose $\eta$ is even and $\varphi N$ is an integer. Without loss of generality suppose that $p_{m}$ is closer to $p_{m+\varphi N}$ than to $p_{m-\varphi N}$ is. Then let $i=m-\varphi N$.

Now construct the interval $D=\left[p_{m}+p_{m-\frac{\eta}{2}}-p_{m-\varphi N}, p_{m}+p_{m+\frac{\eta}{2}}-p_{m-\varphi N}\right]$. Since this interval is of length $p_{m+\frac{\eta}{2}}-p_{m-\frac{\eta}{2}}$, it is weakly larger than $I$, thus by Assumption 5, it contains the ideal policy of at least one agent. If $p_{m+\varphi N} \in D$, then let $j=m+\varphi N$. If $p_{m+\varphi N} \notin D$, then let $j$ be the left-most agent with an ideal policy in $D$. Since $p_{m+\varphi N}-p_{m}<p_{m}-p_{m-\varphi N}$, it follows that if $p_{m+\varphi N} \notin D$, then $p_{j}>p_{m+\varphi N}$. In either case, $p_{j} \geq p_{m+\varphi N}$ and

$$
\widehat{p}_{i j} \in\left[\frac{p_{m}+p_{m-\frac{\eta}{2}}}{2}, \frac{p_{m}+p_{m+\frac{\eta}{2}}}{2}\right] \subset\left[p_{m-\frac{\eta}{2}}, p_{m+\frac{\eta}{2}}\right],
$$

and thus, the difference in support for the two candidates is less than $\eta$. Then $i$ and $j$, by definition of $\eta$, want to run against each other.

Now we show that no other agent would want to enter the race:

A candidate to the left of $p_{i}$ would get at most $m-\varphi N$ votes, whereas $j$ would get no less than $m-\frac{\eta}{2}$. The ratio of their share of supporters is $\frac{\frac{1-2 \varphi}{2} N}{\frac{1}{2} N-\frac{1}{2} \eta}=$ $\frac{(1-2 \varphi) N}{N-\eta}=\frac{1-2 \varphi}{1-\frac{\eta}{N}}$ which converges to $1-2 \varphi<1$, since $\frac{\eta}{N}$ converges to zero. Given a fixed ratio of support between two candidates, the probability that the candidate with the lesser support wins converges to zero. So a candidate to the left of $i$ will not win if $N$ is sufficiently large. $c>c^{*}$ guarantees that sure losers don't run.

Similarly for a candidate that tried to outflank $j$ instead.

Now, a centrist candidate between $i$ and $j$ will collect at most the support of all citizens between $p_{i}$ and $p_{j}$, that is, $2 \varphi N$ if $j=p_{m+\varphi N}$ or $\varphi N$ and all the votes of citizens with ideal policies in $\left(p_{m}, p_{m}+p_{m-\frac{\eta}{2}}-p_{m-\varphi N}\right]$ otherwise. But $\left(p_{m}, p_{m}+p_{m-\frac{\eta}{2}}-p_{m-\varphi N}\right] \subset\left[p_{m}, 2 p_{m}-p_{m-\varphi N}\right]$. By Assumption 6,

$$
2 p_{m}-p_{m-\varphi N}=p_{m}+p_{m}-p_{m-\varphi N}<p_{m}+p_{N-3 \varphi N}-p_{m}=p_{N-3 \varphi N}
$$

and thus there are no more than $\frac{N}{2}-3 \varphi N$ agents with an ideal policy in $\left(p_{m}, p_{m}+p_{m-\frac{\eta}{2}}-p_{m-\varphi N}\right]$. So the centrist entrant gathers a total number of supports which is less than $\frac{N}{2}-2 \varphi N$. Since $i$ gathers at least $\frac{N}{2}-\varphi N$ supporters, their ratio of support is $\frac{1-4 \varphi}{1-2 \varphi}$ which is less than 1 , so the probability that the centrist entrant wins converges to zero. Then $c>c^{*}$ guarantees that the entrant doesn't run. 


\subsection{Lemma 6}

Proof. By contradiction. Suppose $i$ is a spoiler and $i$ is the left-most candidate and let the second left-most candidate be $j$. If every citizen chooses support sincerely and $i$ drops out of the race, all the support for $i$ switches to support for $j$. The probability that $i$ wins the election is now zero, the probability that $j$ wins increases, and the probability that any of the other candidates wins is reduced. Since $v_{i}\left(p_{j}\right) \geq v_{i}\left(p_{k}\right)$ for any candidate $k \notin\{i, j\}$, it follows $\sum_{k \in C \backslash i} v_{i}\left(p_{k}\right) \frac{\operatorname{Pr}\left[W_{k}=1 \mid C\right]}{\operatorname{Pr}\left[W_{i}=0 \mid C\right]} \leq \sum_{h \in C \backslash i} v_{i}\left(p_{h}\right) \operatorname{Pr}\left[W_{h}=1 \mid C \backslash i\right]$.

Since $i$ is a spoiler,

$$
\left\{b+v_{i}\left(p_{i}\right)-\sum_{k \in C \backslash i} v_{i}\left(p_{k}\right) \operatorname{Pr}\left[W_{k}=1 \mid C \backslash i\right]\right\} \operatorname{Pr}\left[W_{i}=1 \mid C\right]<c .
$$

Thus:

$$
\begin{aligned}
\operatorname{Pr}\left[W_{i}\right. & =1 \mid C]\left\{b+v_{i}\left(p_{i}\right)-\sum_{k \in C \backslash i} v_{i}\left(p_{k}\right) \operatorname{Pr}\left[W_{k}=1 \mid C \backslash i\right]\right\} \\
+\operatorname{Pr}\left[W_{i}\right. & =0 \mid C]\left\{\sum_{k \in C \backslash i} v_{i}\left(p_{k}\right) \frac{\operatorname{Pr}\left[W_{k}=1 \mid C\right]}{\operatorname{Pr}\left[W_{i}=0 \mid C\right]}-\sum_{k \in C \backslash i} v_{i}\left(p_{k}\right) \operatorname{Pr}\left[W_{k}=1 \mid C \backslash i\right]\right\}<c .
\end{aligned}
$$

But the left term in the last inequality is just another form of writing the left term in the inequality in condition (i) in Remark 5, which ought to be bigger than $c$ in order for $i$ to be willing to run, thus $i$ cannot be part of an equilibrium with sincere support behavior, thus it cannot be part of a Sincere-Strategic equilibrium.

\subsection{Proposition 8}

Proof. For two candidates: If $i, j \in C$ have different support, it must be they have different ideal policies. Then, given Assumption 4 and Euclidean preferences, every citizen supports either $i$ or $j$ (no indifference). Suppose $S_{j} \leq$ $(1-\epsilon) S_{i}$. Then $S_{i} \geq \frac{1}{2-\epsilon} N$ and $S_{j} \leq \frac{1-\epsilon}{2-\epsilon} N$. Note that $V_{l}$ follows a binomial distribution $B i\left[S_{l}, 1-\mu\right]$ for $l=i, j$. By the Weak Law of Large Numbers, $\frac{V_{i}}{S_{i}}$ and $\frac{V_{j}}{S_{j}}$ converge to $1-\mu$. Therefore, $\frac{V_{i}}{V_{j}}$ converges to $\frac{S_{i}}{S_{j}}$, which is by assumption more than one. Therefore, with probability converging to one as $N$ approaches infinity, $i$ receives more valid votes and wins the election. For a sufficiently low probability of victory, $j$ prefers not to run as candidate.

For three candidates: Let $C=\{i, j, h\}$, where $S_{i} \geq S_{j} \geq S_{h}$. Suppose $S_{h} \leq S_{i}(1-\epsilon)$. By the previous argument with two candidates, the probability that $h$ wins converges to zero. Thus for a large enough $N, h$ has to be a spoiler. By Lemma 1 , it must then be that $p_{h} \in\left(p_{i}, p_{j}\right)$ where both $i$ and $j$ are competitive candidates. In order for $j$ to be competitive, $j$ must win with some positive probability $\gamma^{16}$. Since $V_{i}$ converges to $(1-\mu) S_{i}$ and $V_{j}$ converges

\footnotetext{
${ }^{16}$ We can derive the exact expression of $\gamma$ from Definition 3, but we don't need it for the proof.
} 
to $(1-\mu) S_{j}$, it must then be that $S_{j}$ is itself converging to $S_{i}$ as $N$ approaches infinity. We next show that the probability that given that two candidates tie for victory one of the two is $h$ converges to zero. Let $\delta$ be an arbitrarily small, positive number such that $\delta<\frac{\epsilon}{2}$. Let $\Delta=[1-\delta, 1+\delta]$

The probability that $h$ ties for victory or wins by one vote is:

$$
\begin{aligned}
& \operatorname{Pr}\left[\frac{V_{h}}{V_{i}} \in \Delta\right] \operatorname{Pr}\left[V_{h}-V_{i} \in\{0,1\} \mid \frac{V_{h}}{V_{i}} \in \Delta\right] \operatorname{Pr}\left[V_{h}>V_{j} \mid V_{h}-V_{i} \in\{0,1\}\right] \\
+ & \operatorname{Pr}\left[\frac{V_{h}}{V_{j}} \in \Delta\right] \operatorname{Pr}\left[V_{h}-V_{j} \in\{0,1\} \mid \frac{V_{h}}{V_{j}} \in \Delta\right] \operatorname{Pr}\left[V_{h}>V_{i} \mid V_{h}-V_{j} \in\{0,1\}\right] .
\end{aligned}
$$

The probability that $i$ and $j$ tie for victory is:

$$
\operatorname{Pr}\left[\frac{V_{j}}{V_{i}} \in \Delta\right] \operatorname{Pr}\left[V_{j}=V_{i} \mid \frac{V_{j}}{V_{i}} \in \Delta\right] \operatorname{Pr}\left[V_{j}>V_{h} \mid V_{j}=V_{h}\right] .
$$

Let $\Lambda_{1}=\operatorname{Pr}\left[\frac{V_{h}}{V_{i}} \in \Delta\right] \operatorname{Pr}\left[V_{h}>V_{j} \mid V_{h}-V_{i} \in\{0,1\}\right]$, let $\Lambda_{2}=\operatorname{Pr}\left[\frac{V_{h}}{V_{j}} \in \Delta\right] \operatorname{Pr}\left[V_{h}>\right.$ $\left.V_{i} \mid V_{h}-V_{j} \in\{0,1\}\right]$, let $\Lambda_{3}=\operatorname{Pr}\left[\frac{V_{j}}{V_{i}} \in \Delta\right] \operatorname{Pr}\left[V_{j}>V_{h} \mid V_{j}=V_{h}\right]$. Using this notational shortcut, the ratio of the probability that $h$ ties for victory over the probability that $i$ and $j$ tie for victory is:

$$
\frac{\Lambda_{1} \operatorname{Pr}\left[V_{h}-V_{i} \in\{0,1\} \mid \frac{V_{h}}{V_{i}} \in \Delta\right]+\Lambda_{2} \operatorname{Pr}\left[V_{h}-V_{j} \in\{0,1\} \mid \frac{V_{h}}{V_{j}} \in \Delta\right]}{\Lambda_{3} \operatorname{Pr}\left[V_{j}=V_{i} \mid \frac{V_{j}}{V_{i}} \in \Delta\right]} .
$$

Since $\Lambda_{1}$ and $\Lambda_{2}$ converge to zero and $\Lambda_{3}$ converges to one as $N$ approaches infinity, and

$$
\frac{\operatorname{Pr}\left[V_{h}-V_{i} \in\{0,1\} \mid \frac{V_{h}}{V_{i}} \in \Delta\right]}{\operatorname{Pr}\left[V_{j}=V_{i} \mid \frac{V_{j}}{V_{i}} \in \Delta\right]} \text { and } \frac{\operatorname{Pr}\left[V_{h}-V_{j} \in\{0,1\} \mid \frac{V_{h}}{V_{j}} \in \Delta\right]}{\operatorname{Pr}\left[V_{j}=V_{i} \mid \frac{V_{j}}{V_{i}} \in \Delta\right]}
$$

are bounded, the previous ratio converges to zero. Therefore, given that a single vote is decisive, the probability that it is decisive to determine whether $i$ or $j$ wins converges to one, and for a large enough $N$, supporting $h$ is wasted support. Only citizens whose ideal policy is at a very small distance from $\widehat{p}_{i j}$ will support $h$. Let $d$ be such that any citizen with an ideal policy at a distance more than $d$ from $\widehat{p}_{i j}$ would strategically support $i$ or $j$, not $h$. As $N$ approaches infinity, this distance $d$ converges to zero, the ideal policy of an $h$ supporter has to converge to $\widehat{p}_{i j}$. Let $q^{\prime}$ and $q$ " be the two points in $Q$ closest to $\frac{p_{i}+p_{j}}{2}$ such that $q^{\prime}<\frac{p_{i}+p_{j}}{2}<q^{\prime \prime}$. If $N$ is large enough, $\left[\widehat{p}_{i j}-d, \widehat{p}_{i j}+d\right] \in\left(q^{\prime}, q^{\prime \prime}\right)$ and no citizen will support $h$. Then, by the Minimal Support assumption, $h$ prefers to drop out than to run with no supporters.

For more than three candidates: Suppose first that there are two competitive candidates with distinct ideal policies ${ }^{17}$. If the electorate is sufficiently large,

\footnotetext{
${ }^{17}$ There cannot be an equilibrium in which two or more candidates have a common ideal policy and one other candidate doesn't, because if so strategic citizens would concentrate their support in only one of the candidates with the same policy, and the others would drop out if they had no supporters.
} 
any candidate with support less than $(1-\epsilon)$ times the support of the strongest candidate will have to be a spoiler between the two competitive candidates (Corollary 7), and the probability that a single vote for the spoiler affects the election given that a single vote is pivotal converges to zero. Therefore, by the same arguments as with three candidates, only citizens who are indifferent or close to indifferent about the two competitive candidates would support the spoiler. Eventually, there are no citizens with an ideal policy so close to the midpoint of the ideal policies of the competitive candidates, and then the spoiler who receives no votes drops out of the race. Suppose there are three or more competitive candidates and a weak candidate with support less than $(1-\epsilon)$ times the support of the strongest candidate. With Euclidean policies, no citizen can be simultaneously indifferent about three distinct policies. If the size of the electorate is large enough, the probability that a vote for the weakest candidate is decisive given that a vote is decisive is arbitrarily close to zero. Then, no citizen will support the weakest candidate, and this candidate will drop out, again by the Minimal Support assumption.

\section{References}

[1] Alvarez, M. and J. Nagler, 2000, "A New Approach for Modelling Strategic Voting in Multiparty Elections," British Journal of Political Science, 30, 57-75.

[2] Besley, T., and S. Coate, 1997, "An Economic Model of Representative Democracy," Quarterly Journal of Economics, 112, 85-114.

[3] Eguia, J. 2003, "Is the Status Quo Relevant in a Representative Democracy?" Caltech SS Working Paper 1176.

[4] Lee, D., E. Moretti and M. Butler, 2004. "Do Voters Affect Or Elect Policies? Evidence from the U. S. House," The Quarterly Journal of Economics, $119,807-859$.

[5] Myerson, R. 1993, "Effectiveness of Electoral Systems for Reducing Government Corruption: A Game-Theoretic Analysis," Games and Economic Behavior, 5, 118-132.

[6] Osborne, M.J. and A. Slivinski, 1996, "A Model of Political Competition with Citizen Candidates," Quarterly Journal of Economics, 111, 65-96.

[7] Poole, K. and H. Rosenthal, 1985, "A Spatial Model for Roll Call Analysis," American Journal Political Science, 29, 331-347.

[8] Poole, K. 2005, Spatial Models of Parliamentary Voting, New York, Cambridge University Press.

[9] Riviere, A. 2000, "Citizen Candidacy, Party Formation and Duverger's Law," Discussion Paper dpe00/1, Royal Holloway, University of London. 
[10] Roemer, J. 2003, "Indeterminacy of Citizen-Candidate Equilibrium," Cowles Foundation Discussion Paper No. 1410. 